\title{
Electroproduction of strangeness above the resonance region.
}

\author{
M. Guidal ${ }^{a}$, J.-M. Laget ${ }^{b}$ and M. Vanderhaeghen ${ }^{c}$ \\ ${ }^{a}$ IPN Orsay, F-91406 Orsay, France \\ ${ }^{b}$ CEA/Saclay, DAPNIA/SPhN, F-91191 Gif-sur-Yvette Cedex, France \\ c University Mainz, D-55099 Mainz, Germany
}

(August 1, 2018)

A simple and elegant model, based on Reggeized $t$-channel exchanges is successful in reproducing the main features of all existing data of the reactions $e p \rightarrow e^{\prime} K^{+} \Lambda$ and $e p \rightarrow e^{\prime} K^{+} \Sigma$. In particular, the original way gauge invariance is taken into account is found to be essential to describe the ratio between the Coulomb and the transverse cross-sections at large $Q^{2}$ that has been measured recently at JLab. 
Strangeness production is undergoing a renewed interest in view of the numerous data which are currently coming out of electron accelerators like CEBAF at Jefferson Lab and ELSA at Bonn. It offers us with an original way to probe hadronic matter. Not only the study of the Hyperon-Nucleon interaction is a mandatory complement to the study of the Nucleon-Nucleon interaction, but the implantation of an impurity (the strange quark or a hyperon) in a hadronic system is a formidable tool to study its properties. However, the elementary processes of photo- and electroproduction of kaons off the nucleon should be mastered. In particular, we will show that the determination of the kaon form factor depends on the model used.

At low energy, within about $1 \mathrm{GeV}$ above threshold, many resonances may contribute in the $s$-channel and fits to the scarce data are generally obtained at the expense of many free parameters [1], [2]. At higher energy, Regge phenomenology provides us with an elegant and simple way to account for the analyticity and unitarity of the amplitude with almost no free parameters [3], 沺.

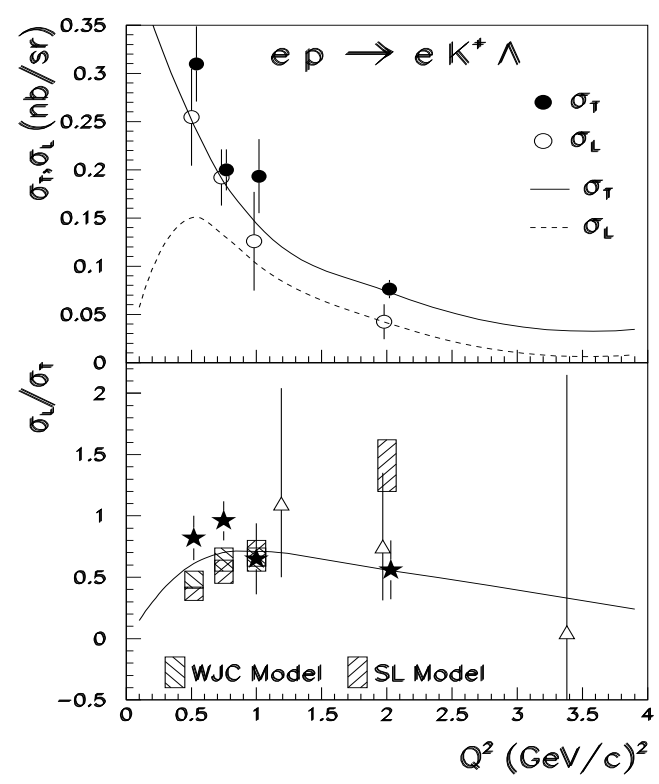

FIG. 1. $Q^{2}$ dependence of $\sigma_{T}$ and $\sigma_{L}$ (upper plot) and $\sigma_{L} / \sigma_{T}$ (lower plot) for the $\gamma^{*}+p \rightarrow K^{+}+\Lambda$ reaction, at $\mathrm{W}=1.84 \mathrm{GeV}$. Experimental data points are from : $(\bullet, \circ$, $\star):[6$ and $(\triangle):$ : 7 . Besides our calculation, on the lower panel are also shown the models of Ref. 11, 2]. The WJC prediction is $\approx 3.5$ (off scale) for $Q^{2}=2.0(\mathrm{GeV} / \mathrm{c})^{2}$.

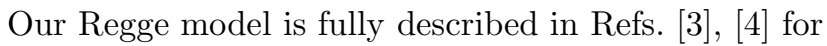
the photoproduction of pions and kaons on the nucleon above the resonance region (above $E_{\gamma} \approx 2 \mathrm{GeV}$ ), and in Ref. [5] for the electroproduction of pions. It is based simply on two "Reggeized" $t$-channel exchanges $(\pi$ and $\rho$ trajectories for pion production, $K$ and $K^{*}$ trajectories for kaon production). An original and essential feature of this model is the way gauge invariance is restored for the $\pi(K) t$-channel exchanges by proper "reggeization" of the $s$-channel nucleon pole contribution. This was found to be the key element to describe numerous features of the experimental data (for instance, the $\pi^{+} / \pi^{-}$ratio, the forward peaking of the charged pion differential cross section, the photon asymmetry, etc...).

As in Ref. [5], we extend the model of kaon photoproduction to electroproduction by multiplying the separately gauge invariant $K$ and $K^{*} t$-channel diagrams by a monopole form factor :

$$
F_{K, K^{*}}\left(Q^{2}\right)=\left[1+Q^{2} / \Lambda_{K, K^{*}}^{2}\right]^{-1},
$$

with $Q^{2}=-q^{2}$, where $q$ is the spacelike virtual photon four-momentum. The mass scales $\Lambda_{K}$ and $\Lambda_{K^{*}}$ are chosen to be $\Lambda_{K}^{2}=\Lambda_{K^{*}}^{2}=1.5 \mathrm{GeV}^{2}$, in order to fit the high $Q^{2}$ behavior of $\sigma_{L}$ and $\sigma_{T}$ in Fig. 1. We keep the same coupling constants at the $(K,(\Lambda, \Sigma), N)$ and $\left(K^{*},(\Lambda, \Sigma), N\right)$ vertices as in the photoproduction study 牧, which were found to describe all existing high energy data, i.e. :

$$
\begin{aligned}
& \frac{g_{K \Lambda N}^{2}}{4 \pi}=10.6, g_{K^{*} \Lambda N}=-23.0, \kappa_{K^{*} \Lambda N}=2.5, \\
& \frac{g_{K \Sigma N}^{2}}{4 \pi}=1.6 \quad, g_{K^{*} \Sigma N}=-25.0, \kappa_{K^{*} \Sigma N}=-1.0
\end{aligned}
$$

The magnitudes and signs $\left(g_{K \Lambda N}<0\right.$ and $\left.g_{K \Sigma N}>0\right)$ of the $K$ strong coupling constants are in agreement with $\mathrm{SU}(3)$ constraints (broken to about 20\%). The signs of the $K^{*}$ strong coupling constants are also in accordance with $\mathrm{SU}(3)$.

It turns out that the recent measurement [6], at Jefferson Lab, of the ratio between the Coulomb $\left(\sigma_{L}\right)$ and the transverse $\left(\sigma_{T}\right)$ cross-sections of the $p\left(e, e^{\prime} K^{+}\right) \Lambda$ reaction clearly favors the Regge model over the resonance models, already in the CEBAF energy range. Fig. 11 shows the comparison of the Regge model with the data. Particularly interesting is the behavior at large $Q^{2}$ of the $\sigma_{L} / \sigma_{T}$ ratio which decreases as the data, in contrast to two recent theoretical resonance models [1], [2]. Our Regge model suitably reproduces the trend of this ratio. The reason is that, due to gauge invariance, the $t$-channel kaon exchange and the $s$-channel nucleon pole terms are indissociable and must be treated on the same footing. In our model [3], 四, they are Reggeized in the same way and multiplied by the same electromagnetic form factor. This approach clearly differs from traditional ones [i], 22 where a different electromagnetic form factor is assigned to the $t$ - and $s$-channel diagrams (monopole and a dipole forms respectively -with also different mass scales, in general). This explicitly breaks gauge invariance and 
the introduction of a purely phenomenological and adhoc counter-term is needed to restore it. Whatever particular way this procedure has been done in the existing literature, it produces a -linearly- rising ratio $\sigma_{L} / \sigma_{T}$ with $Q^{2}$ in contrast to the data. It is important to note that the $Q^{2}$ dependence of this ratio is relatively insensitive to the particular shape or mass scale taken for the electromagnetic form factors of the $K$ and $K^{*}$ and that the decreasing trend observed here is intrinsically linked to the assignment of the same electromagnetic form factor to the $t$ - and $s$-channel Born diagrams (which is clearly the simplest way in fact to keep gauge invariance in electroproduction).

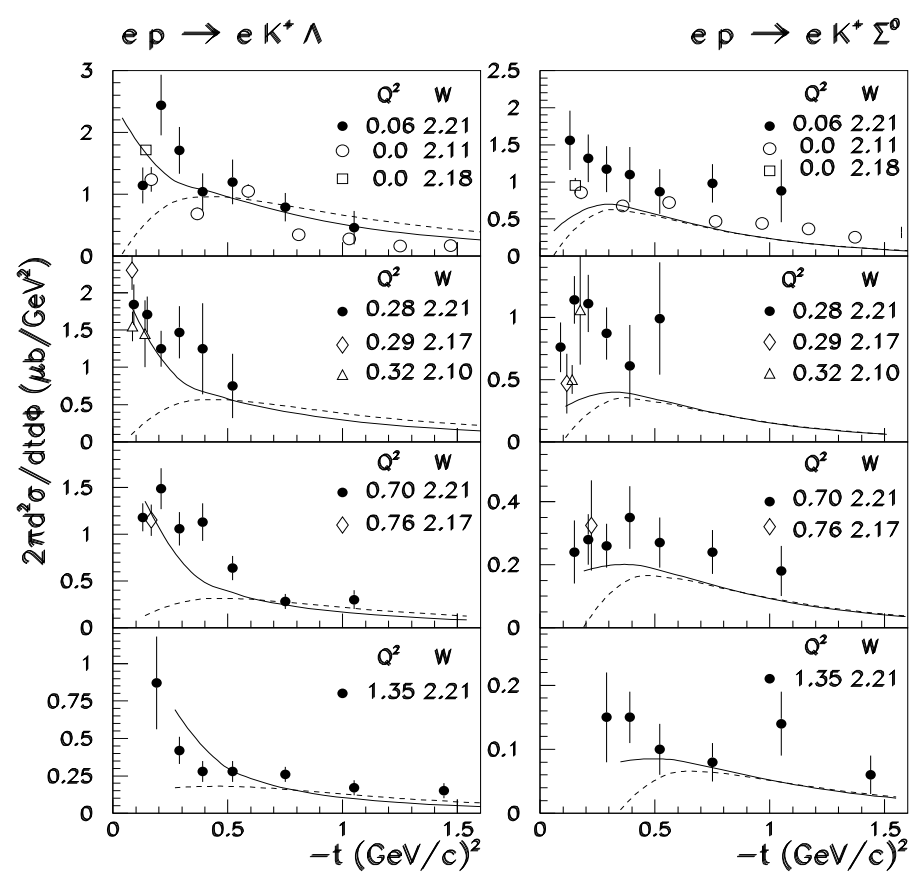

FIG. 2. $t$ dependence of the $\gamma^{*}+p \rightarrow K^{+}+\Lambda$ (left) and $\gamma^{*}+p \rightarrow K^{+}+\Sigma^{0}$ (right) differential electroproduction cross section $2 \pi d^{2} \sigma / d t d \Phi$ for different $Q^{2}$ values. The solid line shows the $K+K^{*}$ exchanges, the dashed line shows the $K^{*}$ exchange only. Displayed data correspond approximately to the same $W$ and $Q^{2}$ ranges (units for the figure are in $\mathrm{GeV} / \mathrm{c}$ and $(\mathrm{GeV} / \mathrm{c})^{2}$ respectively ) ; $(\bullet):$ 8, (०) : [9, (口) : [10, $(\diamond):[11,(\triangle):[12$.

The Regge model reproduces also fairly well the scarce- data prior to Jefferson Lab. Fig. 2 shows the $t$ dependence of the $\gamma^{*}+p \rightarrow K^{+}+\Lambda$ and $\gamma^{*}+p \rightarrow K^{+}+\Sigma^{0}$ differential electroproduction cross section $2 \pi d^{2} \sigma / d t d \Phi$ for different $Q^{2}$ values. The latest [9] and older [10] Bonn data for photoproduction are also shown for reference. At $Q^{2}=0.06 \mathrm{GeV}^{2}$, there is essentially no influence of the form factors. Therefore, without any additional parameter, a straightforward extension of the photoproduction model gives the correct $t$-dependence and magnitude of the data. As in the photoproduction study, the $\Lambda$ and $\Sigma$ channels show a different behavior at forward angles : the differential cross section decreases towards 0 for the latter one whereas it tends to peak for the former one. According to [4, this "peaking" for the $\Lambda$ channel is due to the dominance of the gauge invariant $K$ exchange at small $t$. Because of the weaker $g_{K \Sigma N}$ coupling constant relative to the $g_{K \Lambda N}$ coupling constant, the $K^{*}$ exchange contribution -which has to vanish at forward angles due to angular momentum conservation- dominates the $\Sigma$ channel, which reflects into a decrease of the differential cross section at forward angles. This decrease at small $t$ is attenuated at larger $Q^{2}$ due to the "shift" of $t_{\min }$ with $Q^{2}$.

The Brauel et al. data of Fig. 2 were integrated in $\Phi$ between $120^{\circ}$ and $240^{\circ}$ [8]; so was the model in order to correctly take into account the influence of the $\sigma_{T T}$ and $\sigma_{T L}$ terms which is found not to be negligible. Fig. 2 shows furthermore the destructive interference between the $K$ and $K^{*}$ exchange mechanisms for the $\Sigma$ channel found at large angles, which was also noticed before in the photoproduction study 顿.

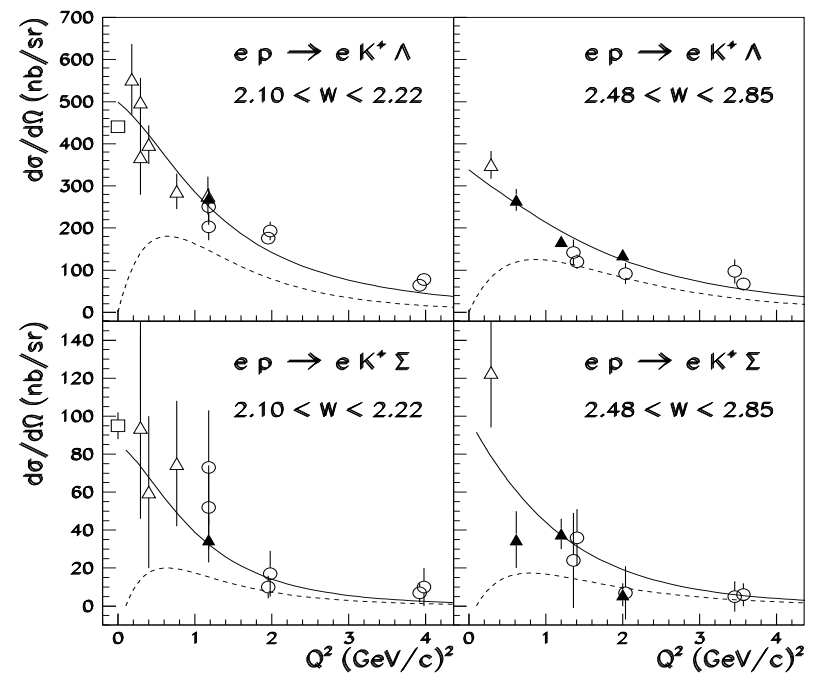

FIG. 3. $Q^{2}$ dependence of the $\gamma^{*}+p \rightarrow K^{+}+\Lambda$ (upper plots) and $\gamma^{*}+p \rightarrow K^{+}+\Sigma^{0}$ (lower plots) differential cross section $d \sigma / d \Omega$ for 2 energy bins. Solid lines : $d \sigma_{T+L} / d \Omega$, dashed line : $d \sigma_{L} / d \Omega$. Displayed data correspond approximately to the same $W$ and $\theta$ ranges ; $(\triangle):$ [13, (o) : [1], $(\Delta):$ 14], ( $\square):$ 10]. This latter point has been renormalized (see text for details).

This $Q^{2}$ dependence is confirmed by Fig. 3 which shows 
the differential cross section $d \sigma / d \Omega$ at $\theta_{c . m .}=8^{\circ}$ as a function of $Q^{2}$ for 2 energy bins. In fact, commonly, this observable has been plotted at a single averaged $W$ value $(<W>=2.15 \mathrm{GeV})$ where a $\left|p_{K}^{*}\right| / W /\left(s-m_{p}^{2}\right)$ dependence was used for the extrapolation of the lower and higher measured $W$ values [11]. Fig. 3 shows that this procedure is approximately right for the $\Lambda$ channel which shows roughly a $\frac{1}{s}$ behavior, but is not appropriate for the $\Sigma$ channel which shows a rather constant behavior in this energy domain. Indeed, it is well known that a Regge amplitude proportional to $s^{\alpha(t)}$ leads to a differential crosssection $\frac{d \sigma}{d t} \propto s^{2 \alpha(t)-2}$ and therefore $\frac{d \sigma}{d \Omega} \propto s^{2 \alpha(t)-1}$. For a $K$-meson exchange dominated mechanism (such as the $\Lambda$ channel at forward angles, see Fig. 2) with $\alpha_{K}(0) \approx$ -0.17 , this implies $\frac{d \sigma}{d \Omega} \propto s^{-1.34}$. And for a $K^{*}$-meson exchange dominated mechanism (such as the $\Sigma$ channel) with $\alpha_{K^{*}}(0) \approx 0.25$, we have $\frac{d \sigma}{d \Omega} \propto s^{-0.5}$.

Note that the photoproduction point of 10 has been renormalized. This data was taken at $\theta_{c . m .}=25^{\circ}(t \approx-0.15$ $\left.\mathrm{GeV}^{2}\right)$. It has been extrapolated to $8^{\circ}\left(t \approx-0.06 \mathrm{GeV}^{2}\right)$ to allow a consistent comparison with the other data. We did so by using the $t$-dependence of our model. From Fig. 2, it can be seen that this implies an upscaling (of $\approx$ 1.2) of the $\Lambda$ photoproduction point and a downscaling ( of $\approx 2.4$ ) of the $\Sigma$ photoproduction point. This leads to a very different figure and conclusion than in Ref. [11] (see Figs. 6 and 7 of Ref. 11]) : firstly, the $Q^{2}$ dependence of the $\Sigma$ channel is not steeper than for the $\Lambda$ channel and, secondly, there is no particular evidence of a rise with $Q^{2}$ of the $\Lambda$ cross-section which would have indicated a strong contribution of $\sigma_{L}$. This latter contribution is seen to account for less than half of the cross section.

It is remarkable that the value of the cut-off mass, $\Lambda_{K}^{2}=\Lambda_{K^{*}}^{2}=1.5 \mathrm{GeV}^{2}$, deduced from the Jefferson Lab experiment leads also to a correct $Q^{2}$ dependence for the world set of previous data, both in the $\Lambda$ and the $\Sigma$ channels. It appears, however, to be quite large, resulting in a rather flat form factor. Indeed, the effective charge radius corresponding to $\Lambda_{K}^{2}=1.5 \mathrm{GeV}^{2}$ is : $<r_{K}^{2}>\equiv-\left.6 \frac{d F_{K}}{d Q^{2}}\right|_{\left(Q^{2}=0\right)}=\frac{6}{\Lambda^{2}} \approx 0.16 \mathrm{fm}^{2}$. This has to be compared to the value measured by direct scattering of kaons on atomic electrons at the CERN SPS which yielded $\left\langle r_{K}^{2}>=0.34 \mathrm{fm}^{2}\right.$ 115. However, we found previoulsy [5] a good agreement between the pion form factor mass scale $\left(\Lambda_{\pi}^{2}=0.462 \mathrm{GeV}^{2}\right.$, i.e. $\left\langle r_{\pi}^{2}>=0.52 \mathrm{fm}^{2}\right)$ deduced from a study similar to the one in this article and the value measured by direct scattering of pions on atomic electrons at the CERN SPS : $\approx 0.44 \mathrm{fm}^{2} 116$. An interpretation of this discrepancy for the kaon case can be that, the kaon pole being far from the physical region, the form factor used in this kind of model does not represent the properties of the kaon itself but rather the properties of the whole trajectory. Instead of being sensitive to the kaon form factor, one might in fact measure a transition form factor between the kaon and an orbital excited state lying on the kaon Regge trajectory. This has to be kept in mind when trying to extract the kaon electromagnetic form factor from these electroproduction reactions.

It is clear that the extracted form factor mass scale depends strongly on the extrapolation from $t_{\min }$ to the pole. Indeed, the standard procedure uses a $\frac{1}{t-m_{K}^{2}}$ dependence reflecting the traditional $t$-channel kaon propagator. However, in our present approach, this propagator is proportional to $s^{\alpha(t)}$ which leads to a steeper (exponential) $t$ dependence. It is therefore no wonder intuitively that the mass scale of the electromagnetic form factor in this latter approach is softer than when using a standard Feynman propagator for the extrapolation.

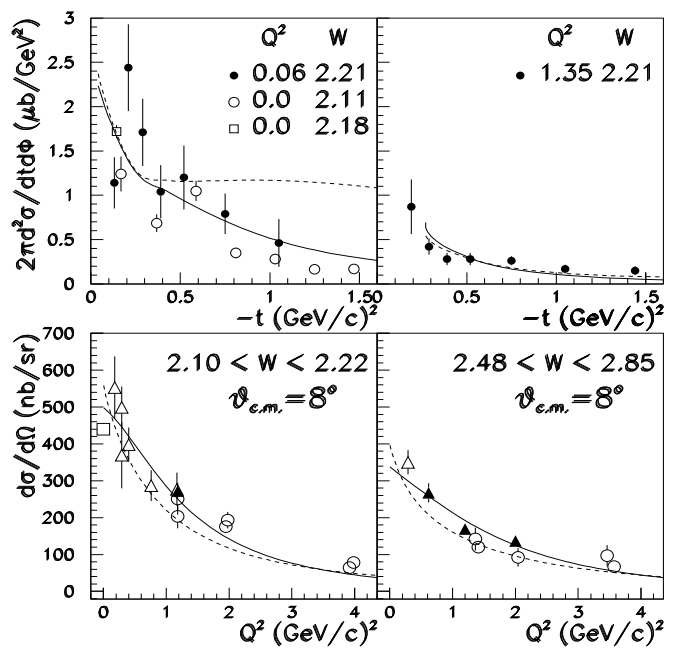

FIG. 4. Comparison of the Regge model -solid lineswith a Born model -dashed lines- (see text for details) for $\gamma^{*}+p \rightarrow K^{+}+\Lambda$. Upper plots : $t$ dependence of the differential cross sections at $Q^{2}=0.0-0.6 \mathrm{GeV}^{2}$ (left) and $Q^{2}=1.35$ $\mathrm{GeV}^{2}$ (right). Lower plots : $Q^{2}$ dependence of the forward differential cross sections for $\langle\mathrm{W}\rangle=2.16 \mathrm{GeV}$ (left) and $<\mathrm{W}>=2.66 \mathrm{GeV}$ (right). Experimental points as in Figs. and 3 .

To understand this crucial point better, we compare on Fig. 1 the two approaches : the plots are extracted from Fig. 2 and 3 and we compare here our Regge model (solid line) and a standard Born model based on the usual $\frac{1}{t-m_{K}^{2}}$ (dashed line). Our Regge model contains $K$ and $K^{*}$ trajectories exchanges but, as can be seen from Fig. 2, only the $K$ trajectory contributes significantly. Our Born model here uses only a (gauge-invariant) $K$ exchange rescaled in order to match the photoproduction result in the forward direction. We left out the $K^{*}$ exchange as it is well known that it diverges with rising energy, due to derivative couplings for exchanged high spin particles. 
The upper left plot of Fig. 6 shows the $t$ dependence of the differential cross section at (almost) the photoproduction point. We see that the Born model based on $K$ exchange alone produces a flatter $t$-dependence at larger $t$ than the Regge model and the data. This could be corrected in principle by introducing an extra hadronic form factor at the $K \Lambda N$ vertex but at the expense of one additional free parameter for the corresponding mass scale. However, this will not give the correct high energy Regge dependence $\left(\frac{d \sigma}{d \Omega} \propto s^{2 \alpha(t)-1}\right.$ and the associated "shrinkage") as was illustrated previously for the pion case [5] where more data are available. A decent $Q^{2}$ dependence for this Born model is obtained with an electromagnetic monopole form factor with $\Lambda_{K}^{2}=0.68$ which is the value exactly corresponding to the kaon charge radius, and, in any case, much smaller than the value needed for the Regge model $\left(\Lambda_{K}^{2}=1.5\right)$. It is clear that it is at small $Q^{2}$ values that one is most sensitive to the mass scale of the electromagnetic form factor as, at larger $Q^{2}$, both form factors show a $\frac{1}{Q^{2}}$ asymptotic behavior. The conclusions here are clear : a traditional Born model (with a $\frac{1}{t-m_{K}^{2}}$ standard Feynman propagator) seems to lead at first sight to a mass scale for the kaon electromagnetic form factor $\left(\Lambda_{K}^{2}=0.68\right)$ compatible with the kaon charge radius. However, such Born model is unable to reproduce the correct energy and $t$ dependences (unless, for this latter case, corrected by an extra hadronic form factor). Furthermore, it is unable to take into account the role of the $K^{*}$ exchange which would diverge (properly taking into account the exchange of higher spin particles was actually one of the main motivations of our Regge model). Let's also notice that a recent direct experimental determination of the proton electric form factor at Jefferson Lab [17] clearly shows that the cut-off mass needed to fit the large $Q^{2}$ data is not consistent with the cut-off mass which is determined by the proton charge radius.

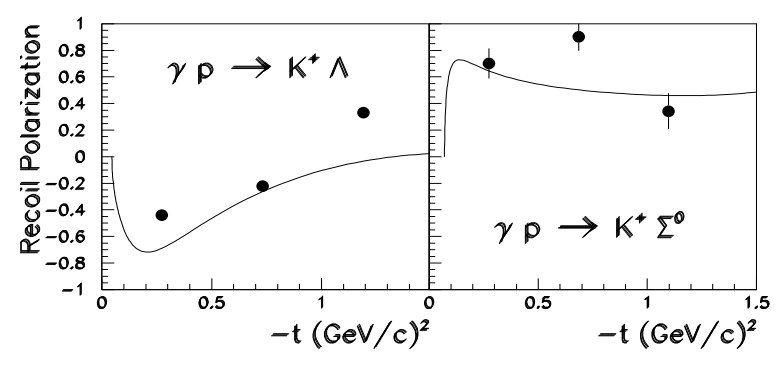

FIG. 5. $t$ dependence of the $\Lambda$ and $\Sigma$ recoil polarizations recently measured at Bonn in photoproduction [9].

We now turn to polarization observables. We first show on Fig. 5 that the $\Lambda$ and $\Sigma$ single recoil polarizations recently measured at Bonn [9] in photoproduction are reasonnably predicted by our model at forward angles (At larger angles, other contribution besides $t$-channel exchanges are expected to contribute : $u$-channel exchanges, resonances contributions at low energies, etc...). It is the interference between the $K$ and $K^{*}$ trajectories -arising from the $e^{i \alpha_{K, K^{*}}(t)}$ signature term in the $K$ and $K^{*}$ Regge propagators, see Ref. [4]- which produces the non-zero polarization in the present model. The negative (positive) $\Lambda(\Sigma)$ recoil polarization is directly related to the relative sign of the $g_{K^{*} N \Lambda}$ couplings with respect to the $K\left(g_{K \Lambda N}<0\right.$ and $g_{K \Sigma N}>0$ whereas $g_{K^{*}(\Lambda, \Sigma) N}$ are both negative).

At Jefferson Lab, with electron beams, double polarization observables will soon be accessed for the first time [18]. These will put further stringent constraints on the models and will allow to disentangle the contributions of the $K$ and the $K^{*}$ exchange. Typical behaviors for kinematics accessible at Jefferson Lab $\left(E_{e}=6 \mathrm{GeV}\right.$, $\theta_{e^{\prime}}=13^{\circ}, E_{\gamma^{*}}=2.643 \mathrm{GeV}$ ) are shown in Fig. 6 for $\Phi=0^{\circ}$ and $180^{\circ}$ (The definitions and the notations are given in Appendix A of Ref. [19], and the $z$-axis is chosen along the direction of the virtual photon). If only one Regge trajectory is retained the induced polarization $P_{Y}^{\circ}$ (unpolarized electrons) vanishes: it is different from zero when the two trajectories interfere. It is worth noting that $P_{Y}^{\circ}$, which is the "extension" to electroproduction of the photoproduction recoil polarization of Fig. 5, is now positive (at $\Phi=0^{\circ}$ ). Indeed, in photoproduction, this observable is sensitive only to $\sigma_{T}^{Y}$ whereas in electroproduction the additionnal $\sigma_{L}^{Y}, \sigma_{T T}^{Y}$ and $\sigma_{T L}^{Y}$ cross sections enter. These latter contributions come with an oppposite sign with respect to $\sigma_{T}^{Y}$ here. The sideways $\left(P_{X}^{\prime}\right)$ and longitudinal $\left(P_{Z}^{\prime}\right)$ transfered polarizations indicate the relative amount of $K$ and $K^{*}$ exchanges. The strong $\Phi$ dependence evidenced on Fig. 6 can also be used to disentangle the various contributions. At large transfers, it was noted that, in Deep Inelastic Scattering, the strange 
quark can be used to follow the spin transfer from the probe to the emitted $\Lambda$. It will be interesting to compare such approaches to our Regge calculations.

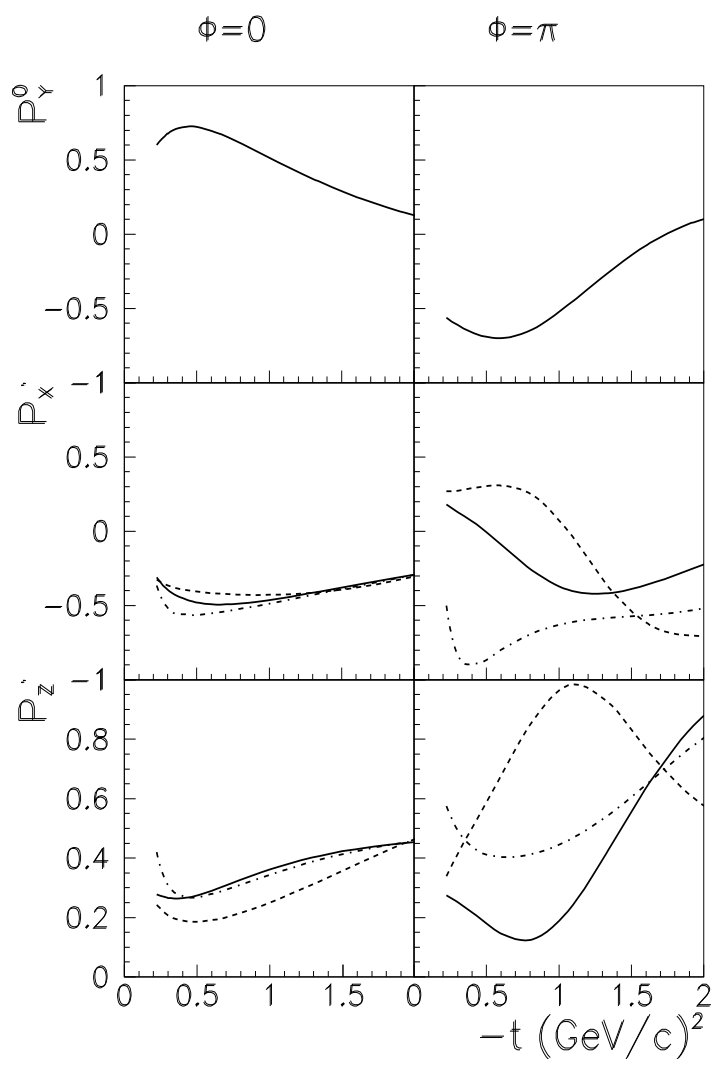

FIG. 6. The $t$ dependence of the three $\Lambda$ polarizations which do not vanish in coplanar kinematics, for $E_{e}=6 \mathrm{GeV}$, $W=2.2 \mathrm{GeV}$ and $Q^{2}=1 \mathrm{GeV}^{2}$. Dashed lines: $K$ exchange only. Dash-dotted lines: $K^{*}$ exchange only. Full lines: complete model. Left panels : $\Phi=0^{\circ}$ degrees, right panels : $\Phi=180^{\circ}$

To conclude, this simple and elegant Regge trajectory exchange model accounts fairly well for the whole set of available data and the rather accurate first measurement at Jefferson Lab. Since the model depends on very few parameters, the forthcoming data from Jefferson Lab will constitute a stringent test (that may eventually call for a more fundamental partonic description). Nevertheless, it provides already a good starting point to compute and analyze strangeness production in nuclei.

We would like to acknowledge useful discussion with P. Bydzovsky. This work was supported in part by the French CNRS/IN2P3, the French Commissariat à l'énergie Atomique and the Deutsche Forschungsgemeinschaft (SFB443).
[1] R.A. Williams, C.R. Ji and S.R. Cotanch, Phys. Rev. C 46, 1617 (1992).

[2] J.C. David, C. Fayard, G.H. Lamot and B. Saghai, Phys. Rev. C 53, 2613 (1996).

[3] M. Guidal, J.-M. Laget and M. Vanderhaeghen, Phys. Lett. B 400, 6. (1997).

[4] M. Guidal, J.-M. Laget and M. Vanderhaeghen, Nucl. Phys. A 627, 645 (1997).

[5] M. Vanderhaeghen, M. Guidal and J.-M. Laget, Phys. Rev. C 57, 1454 (1998).

[6] G. Niculescu et al., Phys. Rev. Lett. 81, 1805 (1998).

[7] C.J. Bebek, C.N. Brown, R.V. Kline, F.M. Pipkin, S.W. Raither, L.K. Sisterson, A. Browman, K.M. Hanson, D. Larson and A. Silverman, Phys. Rev. D 15, 3082 (1977).

[8] P. Brauel, T. Canzler, D. Cords, R. Felst, G. Grindhammer, M. Helm, W.-D. Kollmann, H. Krehbiel and M. Schädlich, Z. Phys. C 3, 101 (1979).

[9] M.Q. Tran et al., Phys. Lett. B 445, 20 (1998).

[10] P. Feller, D. Menze, U. Opara, W. Schulz and W.J. Schwille, Nucl. Phys. B 39, 413 (1972).

[11] C.J. Bebek, C.N. Brown, P. Bucksbaum, M. Herzlinger, S.D. Holmes, C.A. Lichtenstein, F.M. Pipkin, S.W. Raither and L.K. Sisterson, Phys. Rev. D 15, 594 (1977).

[12] T. Azemoon, I. Dammann, C. Driver, D. Lüke, G. Specht, K. Heinloth, H. Ackermann, E. Ganssauge, F. Janata and D. Schmidt, Nucl. Phys. B 95, 77 (1975).

[13] C. N. Brown, C.R. Canizares, W.E. Cooper, A.M. Eisner, G.J. Feldman, C.A. Lichtenstein, L.Litt, W. Lockeretz, V.B. Montana and F.M. Pipkin, Phys. Rev. Lett. 28, 1086 (1972).

[14] C. J. Bebek et al., Phys. Rev. Lett. 32, 21 (1974).

[15] S. R. Amendolia et al., Phys. Lett. B 178, 435 (1986).

[16] S. R. Amendolia et al., Phys. Lett. B 146, 116 (1984).

[17] M. K. Jones et al., nucl-ex/9910005 (1999).

[18] JLab proposals, E99-006, D. Carman et al., E98-101, O. Baker et al. .

[19] J.-M. Laget, Nucl. Phys. A 579, 333 (1994). 